\title{
A New Train Timetable Optimization Model Using a Lagrangian Relaxation Guided Heuristic for a Real-World High-Speed Railway Line
}

\author{
Xuecheng Yan ${ }^{1, a^{*}}$, Yixiang Yue ${ }^{1, b}$ and Deyi $\mathrm{Li}^{1, \mathrm{c}}$ \\ ${ }^{1}$ Beijing Jiaotong University, Beijing, China

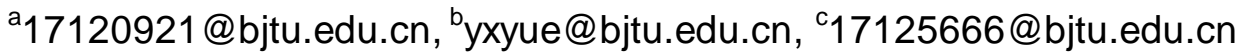

Keywords: Train timetabling; Lagrangian relaxation; Shortest-path problem; Train service plan

\begin{abstract}
High-speed railway (HSR) systems have been developing rapidly in China and other countries throughout the past decade. A high-quality train timetable should satisfy transportation demands with the best possible benefit. This paper presents a scheduling model for double-track HSR lines based on a train service plan for timetable optimization. First, we construct a space-time network and assign each resource a usage cost at every discrete instant of time to maximize the total profit of a train timetable as a whole. Second, we propose a heuristic method based on the Lagrangian relaxation (LR) algorithm to solve the model by updating the resource usage costs according to the conflicts caused by trains in each iteration. Finally, we consider the Beijing-Shanghai HSR line as a real-world application of the methodology. The results show that the proposed model and heuristic offer an efficient and promising means of addressing HSR timetable problems.
\end{abstract}

\section{Introduction}

The train timetabling problem (TTP) has been studied in different ways and from various perspectives over the past four decades $[1,2,3]$ summarized the most up-to-date advanced railway operation techniques. Cacchiani and Toth [4] presented an overview of the major efforts concerning train timetabling. Corman et al. [5] considered the bi-objective problem of minimizing both train delays and missed connections to obtain a set of feasible non-dominated schedules. Several scholars have presented excellent surveys of the available train-timetabling algorithms such as metaheuristic algorithms [6], backtracking search algorithms [7] and look-ahead search algorithms [8].

The research presented in this paper was motivated by the desire to schedule the best timetable for maximizing the total profit while adhering to a given train service plan when different grades of HSR trains operate in the same network. And an important feature of our analysis is that the optimization starts not from blank train working diagrams but rather from a given train service framework.

\section{Train Timetable Model}

Problem Description. Train $j$ departs from the virtual origin node with $\alpha$ choices of possible departure times, and for each node representing its departure from station 1 toward station 2, there are also $\alpha$ choices of times for its subsequent departure from station 2; thus, the maximum number of timetable plans for train $j$ is $\alpha^{\beta}$.

Let us suppose that the running time for all trains of the same class in a given section is constant; to obtain the total travel time, we must add an acceleration and deceleration delay as well as the stop time at the corresponding station.

We consider railway stations as resources on which trains must bid and assign a usage price to each resource in every time unit. The path of a train represented by the train timetable has a corresponding total profit of the train; different operation plans incur different resource usage costs for a given train. When there is a conflict, the conflicting trains must bid for the use of the contested resource, thereby modifying the price at the time of the conflict.

Notation. The general subscripts, input parameters and decision variables used in our mathematical equations are listed as follows: $h, h^{\prime}$ are the segment indices $h, h^{\prime} \in O$, where $O$ is the set of segments, 
$|O|=o .{ }^{i, i^{\prime}}$ are the station indices, $i, i^{\prime} \in S$, where $S$ is the set of stations, $|S|=s . m$ is the track index, $m \in M_{i}$, where $M_{i}$ is the set of sidetracks at station i. ${ }^{i, j^{\prime}}$ are the train indices, $j_{j} j^{\prime} \in T$, where $T$ is the set of trains, $|T|=t .{ }^{k, k^{\prime}, k^{\prime \prime}}$ are the time indices, $k, k^{\prime}, k^{\prime \prime} \in Q$, where $Q$ is the set of timestamps, $|Q|=q$

The binary decision variables included in the programming model are as follows:

$$
\begin{aligned}
x_{i j k} & =\left\{\begin{array}{ll}
1 & \text { if train } j \text { departs from station } i \text { at time } k \\
0 & \text { otherwise }
\end{array} \quad y_{i j k}= \begin{cases}1 & \text { if train } j \text { arrives at station at timk } \\
0 & \text { otherwise }\end{cases} \right. \\
z_{i j k m} & = \begin{cases}1 & \text { if train } j \text { stops on track } m \text { of station } i \text { at time } k \\
0 & \text { otherwise }\end{cases}
\end{aligned}
$$

The arrival time of a train at a station is determined by its departure time from the previous station and its running time in the section between the two stations; the stopping time is computed from the arrival and departure times using Eq. 1 and Eq. 2. And the parameters are as follows: ${ }^{b_{i j k}}$ is the benefit for train $\mathrm{j}$ departing from station $\mathrm{i}$ at time $\mathrm{k}, U_{h}^{o}$ is the first station in segment $\mathrm{h}, V_{h}^{o}$ is the last station in segment $\mathrm{h}, G$ is the set of train grades, $G=\{1,2, \cdots, \mathrm{g}\}$. And $\Phi_{\Phi_{i j}^{\max }}$ is the best time span for train $\mathrm{j}$ to depart from station $\mathrm{i}, \Phi_{i j}$ is the allowed time for train $\mathrm{j}$ at station $\mathrm{i}$ based on the train service plan, $U_{j}^{T}$ is the origin station of train $\mathrm{j}, V_{j}^{T}$ is the terminal station of train $\mathrm{j}, H D$ is the minimum departure headway between two consecutive trains from a station, $H A$ is the minimum arrival headway between two consecutive trains into a station, $R_{i j}$ is the running time for train $\mathrm{j}$ between stations $\mathrm{i}$ and $\mathrm{i}+1, P_{i j}$ is the minimum stopping time for train $\mathrm{j}$ at station $\mathrm{i}$.

The variables associated with timetable decisions are as follows: $d_{i j}$ is the departure time of train $\mathrm{j}$ from station $\mathrm{i}, i \in S, j \in T, a_{i j}$ is the arrival time of train $\mathrm{j}$ at station $\mathrm{i}, i \in S, j \in T . w_{i j}$ is the waiting time of train $\mathrm{j}$ at station $\mathrm{i}, i \in S, j \in T$.

(1) $a_{i^{\prime} j}$ is computed from $d_{i j}$ and $R_{i j}$ :

$a_{i^{\prime} j}=d_{i j}+R_{i j} \quad \forall i, i^{\prime} \in S, i^{\prime}=i+1, j \in T$

(2) $w_{i j}$ is computed from $d_{i j}, a_{i j}$ and $P_{i j}$ :

$$
w_{i j}=d_{i j}-a_{i j}-P_{i j} \quad \forall i \in S, j \in T
$$

$x_{i j k}, y_{i j k}$ and $z_{i j k m}$ are the train schedule decision variables and correspond to $d_{i j}, a_{i j}$ and $w_{i j}$.

Definition of Benefit. According to Eq. 1 and Eq. 2, $a_{i^{\prime} j}$ and $w_{i j}$ can be replaced with expressions in terms of $d_{i j}$. Thus, the benefit function for train $j$ departing from station $i$ can be written as Eq. 3 .

$$
b_{i j k}=\left\{\begin{array}{ll}
b_{i j}^{\max } & k \in \Phi_{i j}^{\max } \\
b_{i j}-\rho \times \delta & k \in \Phi_{i j} \backslash\left\{\Phi_{i j}^{\max }\right\} \\
0 & k \notin \Phi_{i j}
\end{array}\right\}
$$

$b_{i j}^{\max }$ is the maximum benefit value of train $i$ departing from station $i, b_{i j k}$ is the actual benefit, $\Phi_{i j}^{\max }$ is the set of best departure times based on the train service plan, $\Phi_{i j}$ is the set of all departure times corresponding to the allowed time, and $\delta$ is the absolute difference between the actual train departure time and the best departure time. $\rho$ is the deviation penalty factor, the value of which is determined by the decreasing slope on either side of the set of best departure times.

Mathematical model. The HSR timetable model can be formulated as follows: The objective function is:

$$
\max \sum_{i \in S} \sum_{j \in T} \sum_{k \in Q}\left(x_{i j k} \times b_{i j k}\right)
$$

(1) At most, one train can depart from a given station at a given time. 


$$
\sum_{k \in Q} x_{i j k} \leq 1 \quad \forall i \in S, j \in T
$$

At most, one train can arrive at a given station at a given time.

$$
\sum_{k \in Q} y_{i j k} \leq 1 \quad \forall i \in S, j \in T
$$

(2) For each train, the numbers of departure and arrival times are equal.

$$
\sum_{i \in S} \sum_{k \in Q} x_{i j k}=\sum_{i \in S} \sum_{k \in Q} y_{i j k} \quad \forall j \in T
$$

(3) The interval between two consecutive departures from the same station $i$ must be greater than or equal to $H D$. The interval between two consecutive arrivals at the same station i must be greater than or equal to HA.

$$
\begin{aligned}
& \sum_{j \in T} \sum_{k=k^{\prime}}^{k^{\prime}+H D} x_{i j k} \leq 1 \quad \forall i \in S, k^{\prime} \in Q \\
& \sum_{j \in T} \sum_{k=k^{\prime}}^{k^{\prime}+H A} y_{i j k} \leq 1 \quad \forall i \in S, k^{\prime} \in Q
\end{aligned}
$$

(4) At most, only one train can stop on a given sidetrack at station $i$ at a given time.

$$
\sum_{j \in T} z_{i j k m} \leq 1 \quad \forall i \in S, k \in Q, m \in M_{i}
$$

(5) Trains cannot overtake each other in any section. Suppose that train $i$ departs from station $i$ in time unit $k^{\prime}$ and arrives at station in time unit $k^{\prime \prime}$. We select points $k^{\prime}$ and $k^{\prime \prime}$ as reference time units. The overtaking constraint states that a different train $i^{\prime}$ cannot depart from station $i$ before time unit $k^{\prime}$ and also arrive at station $i^{\prime}$ after time unit $k^{\prime \prime}$.

$$
x_{i j k^{\prime}}+y_{i^{\prime} j k^{\prime \prime}}+\sum_{k=1}^{k^{\prime}} x_{i j^{\prime} k}+\sum_{k=k^{\prime \prime}}^{q} y_{i^{\prime} j^{\prime} k} \leq 3 \quad \forall i, i^{\prime} \in S, i^{\prime}=i+1, j, j^{\prime} \in T
$$

The number of decision variables is $3 \times s \times t \times q$. The number of constraints is determined as follows: Eq. 5 and Eq. 6 give rise to $(s-1) \times t$ constraints, Eq. 7 gives rise to $t$ constraints, Eq. 8 and Eq. 9 give rise to $(s-1) \times q$ constraints, Eq.10 gives rise to $s \times q \times \sum_{i} M_{i}$ constraints, and Eq.11 gives rise to $(s-1) \times t \times(t-1) / 2$ constraints.

\section{Lagrangian Relaxation-based Heuristic Algorithm for Solving the Optimization Problem}

Lagrangian relaxation algorithm. We present an LR-based heuristic approach combined with a Dijkstra algorithm to determine the optimal solutions to a set of sub-problems. Given a set of non-negative Lagrange multipliers, the primal problem is denoted by $P_{L R}$ :

$\mathrm{PLR}=\max \mathrm{L}$

$$
L=\sum_{i \in S} \sum_{j \in T} \sum_{k \in Q}\left(x_{i j k} \times b_{i j k}\right)+\sum_{k \in Q} \sum_{i \in S} \lambda_{i k}^{d}\left(1-\sum_{j \in T} x_{i j k}\right)+\sum_{k \in Q} \sum_{i \in S} \lambda_{i k}^{a}\left(1-\sum_{j \in T} y_{i j k}\right)-\sum_{k \in Q} \sum_{i \in S} \lambda_{i k m}^{s}\left(1-\sum_{j \in T} \sum_{m \in M_{i}} z_{i j k m}\right) \text { s.t. (5), (6), (7). }
$$

Consider the following two equations depending on Eq.1 and Eq.2:

$$
\begin{aligned}
& y_{i^{\prime} j k}=x_{i j k^{\prime}} \quad \forall k, k^{\prime} \in Q, k=k^{\prime}+R_{i j}, i^{\prime} i^{\prime} \in S, i^{\prime}=i+1 \\
& \sum_{m \in M_{i}} z_{i j k m} \geq x_{i j k^{\prime \prime}} \quad \forall k, k^{\prime \prime} \in Q, k \in\left[k^{\prime \prime}-P_{i j}-w_{i j}, k "\right], i \in\left(U_{j}^{T}, V_{j}^{T}\right)
\end{aligned}
$$

By replacing $y_{i^{\prime} j k}$ and ${ }^{z_{j k k n}}$ with ${ }^{x_{i j k^{\prime}}}$ and ${ }^{x_{i j k^{n}}}$ using Eq. 13 and Eq. 14 and then simplifying Eq. 12, we obtain a new equation: 


$$
\begin{aligned}
& L \leq \sum_{i \in S} \sum_{j \in T} \sum_{k \in Q}\left(x_{i j k} \times b_{i j k}\right)-\sum_{i \in S} \sum_{j \in T} \sum_{k \in Q}\left(\begin{array}{l}
\lambda_{i k}^{d} \times x_{i j k}+\lambda_{i^{\prime} k^{\prime}}^{a} \times x_{i j k^{\prime}} \\
+\sum_{m \in M_{i}} \lambda_{i k^{\prime} m}^{s} \times x_{i j k}
\end{array}\right)+\sum_{i \in S} \sum_{k \in Q}\left(\lambda_{i k}^{d}+\lambda_{i^{\prime} k^{\prime}}^{a}+\sum_{m \in M_{i}} \lambda_{i k k^{\prime} m}^{s}\right) \\
& i, i^{\prime} \in S \text { and } k, k^{\prime}, k^{\prime \prime} \in Q \text {, and let } \lambda_{i k}=\lambda_{i k}^{d}+\lambda_{i^{\prime} k^{\prime}}^{a}+\sum_{m \in M_{i}} \lambda_{i k^{\prime \prime} m}^{s}, \\
& L^{\prime}=\max L: L^{\prime}=\sum_{i \in S} \sum_{j \in T} \sum_{k \in Q}\left(x_{i j k} \times b_{i j k}\right)-\sum_{i \in S} \sum_{j \in T} \sum_{k \in Q}\left(\lambda_{i k} \times x_{i j k}\right)+\sum_{i \in S} \sum_{k \in Q} \lambda_{i k} \quad \text { s.t. (5),(6),(7). }
\end{aligned}
$$

The Lagrangian dual problem is as follows: $P_{L D}=\min _{\left(\lambda_{i k}\right)} \max L^{\prime}$

$$
L^{\prime}=\sum_{i \in S} \sum_{j \in T} \sum_{k \in Q}\left(x_{i j k} \times b_{i j k}\right)-\sum_{i \in S} \sum_{j \in T} \sum_{k \in Q}\left(\lambda_{i k} \times x_{i j k}\right)+\sum_{i \in S} \sum_{k \in Q} \lambda_{i k} \text { s.t. (5), (6), (7). }
$$

We can simplify Eq. 17 to obtain a new equation:

$$
\begin{aligned}
& \min _{\left(\lambda_{i k}\right)} \max L^{\prime}=\min _{\left(\lambda_{i k}\right)} \max \left[\sum_{j \in T} C_{j}+C_{0}\right] \quad \text { s.t. (5),(6),(7). } \\
& C_{0}=\sum_{i \in S} \sum_{k \in Q} \lambda_{i k} \\
& C_{j}=\sum_{i \in S} \sum_{k \in Q}\left(x_{i j k} \times b_{i j k}-\lambda_{i k} \times x_{i j k}\right)
\end{aligned}
$$

$C_{0}$ is a fixed value corresponding to $\lambda_{i k}$ in each iteration. $C_{j}$ indicates that the train timetable does not consider the conflicts caused by different trains attempting to use the same resources. The Lagrange multipliers $\lambda_{i k}$ represent the prices paid due to competition between trains at time $\mathrm{k}$ at station $\mathrm{i}$. The purpose of Eq. 18 is to maximize the overall profit attained by summing the values of all trains minus the resource usage costs.

Lagrangian Relaxation Sub-Problem. A sub-problem corresponds to a train's path. For each sub-problem, the objective is to find an optimal solution with a maximized profit; in the model $\left(P_{L D}\right), \lambda_{i k}$ is constant in each iteration n. If we obtain every maximum value of $\sum_{j \in T} C_{j}$, then $L$ will also be maximized. The value of $C_{j}$ can be interpreted as the timetable benefit minus the resource usage cost for train $i$. The primal problem can thus be decomposed into many independent sub-problems, where the objective of each sub-problem is to seek an optimal solution for a single train.

We adopt heuristic methods to reduce the search space when obtaining the optimal solutions to the sub-problems. As shown in Fig. 1, and reduce the solutions for each segment to obtain a much smaller solution space. In accordance with Eq. 1 and Eq. 2, we delete solutions based on their departure and arrival times. In each section, $R_{i j}$ and $P_{i j}$ are constants and if $d_{i^{\prime} j}-d_{i j} \leq R_{i j}+P_{i^{\prime} j}\left(\forall i, i^{\prime} \in S, i^{\prime}=i+1, j \in T\right)$ the corresponding solution is forbidden. Using this heuristic, we can obtain a much simpler network for each sub-problem, as shown in Fig. 1. In order to achieve compatibility with the classical shortest-path problem, every arc in the network is weighted as follows: for every virtual arc, the weight is zero, whereas for every feasible solution, the weight of the arc is defined by Eq. 21.

$$
\begin{aligned}
& \pi_{h j}^{f}=\sum_{i=U_{h}^{o}}^{V_{i j}^{o}} b_{\max }-\sum_{i=U_{h}^{o}}^{V_{h}^{o}} \sum_{k \in Q}\left(x_{i j k} \times b_{i j k}-\lambda_{i k} \times x_{i j k}\right) \\
& \pi_{h j}^{f} \text { is the weight of the } f \text {-th arc in segment } h \text { for train } i .
\end{aligned}
$$

The profit for each train is

$$
\begin{aligned}
& \pi_{j}=\sum_{i \in S} b_{i j}^{\max }-\sum_{i \in S} \sum_{k \in Q}\left(x_{i j k} \times b_{i j k}-\lambda_{i k} \times x_{i j k}\right) \\
& \sum_{i \in S} b_{i j}^{\max } \text { is a constant, and } \sum_{i \in S} b_{i j}^{\max } \geq C_{j} .
\end{aligned}
$$

If we are searching for the path with the lowest price $\left(\pi_{j}\right)$ from the virtual start node to the virtual terminal node in a space-time network, then $C_{j}$ is the optimal result. Searching for the optimal solution to a sub-problem requires seeking the shortest path from the virtual start node to the virtual terminal 
node in the network, as shown in Fig. 1. We use a Dijkstra algorithm to solve each sub-problem and rapidly determine the optimal solution.

Lagrange multipliers. There are two major methods of computing Lagrange multipliers: the subgradient method and the bundle method.

(1) Subgradient method: It is a classical method of updating Lagrange multipliers. The Lagrange multipliers are updated using the following equation:

$$
\lambda_{i k}^{n+1}=\lambda_{i k}^{n}-\frac{\gamma_{s}^{n}}{\left\|g\left(\lambda_{i k}^{n}\right)\right\|^{2}} g\left(\lambda_{i k}^{n}\right)
$$

$g\left(\lambda_{i k}^{n}\right)$ is the subgradient at point $(i, k)$ in iteration $\mathrm{n} . \gamma_{s}^{n}$ is the regulatory factor for the step length. The initial value of the multiplier is $\lambda_{i k}^{0}=1$. A smaller value of $\gamma_{s}^{n}$ yields a more accurate result; a larger $\gamma_{s}^{n}$ allows faster convergence but causes the result to be less accurate. Through testing, we must achieve a trade-off between the convergence and accuracy of the algorithm. $\gamma_{s}^{n}$ is updated using Eq. 24:

$$
\gamma_{s}^{n}=\frac{1}{15+n / 12}
$$

2) Bundle method:

$$
\text { Minimize }\left\|\lambda-\lambda_{i k}^{n^{\prime}}\right\| \text {, s.t. } \overline{P_{L D}^{n}} \geq P_{L D}\left(\lambda_{i k}^{n^{\prime}}\right)+g\left(\lambda_{i k}^{n^{\prime}}\right)^{T}\left(\lambda-\lambda_{i k}^{n^{\prime}}\right), \forall n^{\prime}<n, \quad \lambda \geq 0
$$

$g\left(\lambda_{i k}^{n}\right)$ is the subgradient at point $(i, k)$ in iteration n. $\gamma_{b}^{n}$ is the regulatory factor for the step length. In the method described above, the target value $\overline{P_{L D}^{n}}$ is updated according to $\overline{P_{L D}^{n}}=\left(1-\gamma_{b}^{n}\right) \times P_{L D}^{n}+\gamma_{b}^{n} \times P_{L R}$, where $\gamma_{b}^{0}=1$. Through testing, a trade-off between the convergence and accuracy of the algorithm is achieved. $\gamma_{b}^{n}$ is updated using Eq.26:

$$
\gamma_{b}^{n}=\left(\frac{3}{5}\right)^{n}
$$

Solution procedure. The complete solution procedure using the proposed heuristic approach is shown in Fig. 2.

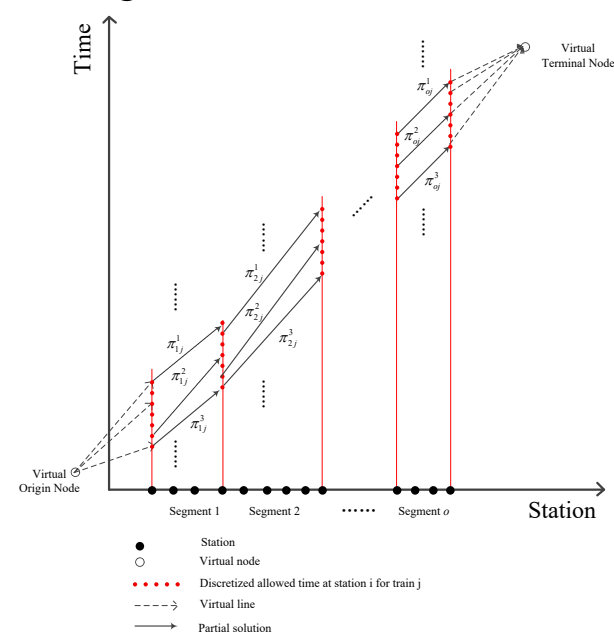

Figure 1. Illustration of a shortest-path problem

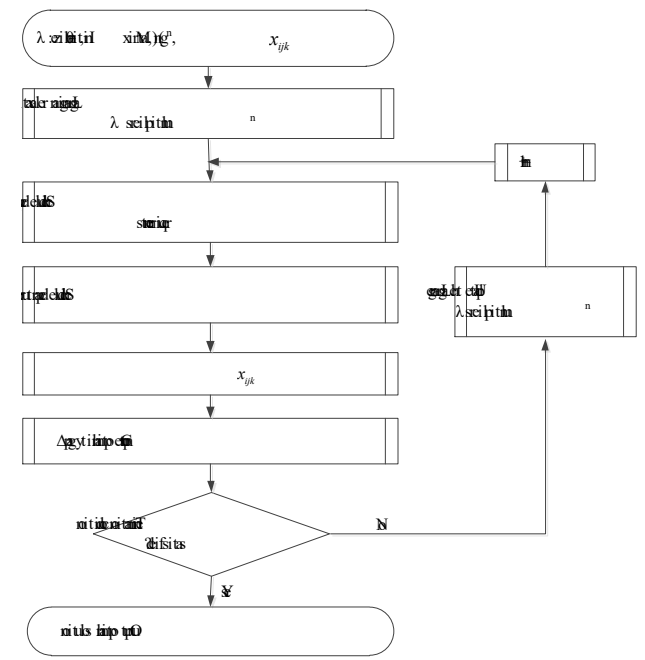

Figure 2. Procedure for applying the solution methodology

\section{Case study}

Beijing-Shanghai high-speed railway. With a total length of 1,302 kilometers, this HSR system consists of 23 stations. We designate seven stations (Beijing Nan Station, Tianjin Station, Jinan Xi 
Station, Xuzhou Dong Station, Bengbu Nan Station, Nanjing Nan Station, and Shanghai Hongqiao Station) as nodes, thereby defining six segments of the line. And we know that each station's name, number of tracks, distance from the Beijing Nan Station, the running times in each section for different grades of trains and the number of different grades of trains travel at different speeds [9].

The scheduling algorithms were implemented in Microsoft Visual Studio 2010 on the Windows 7 operating system, and CPLEX Optimization Studio was used as the linear programming solver.

Results and analysis. We obtained the results after 80 iterations.

Table 4 Adjustment Results for Different Cases of Train Operations

\begin{tabular}{|c|c|c|c|c|c|c|}
\hline $\begin{array}{c}\text { Method of } \\
\text { updating } \\
\begin{array}{c}\text { Lagrange } \\
\text { multipliers }\end{array}\end{array}$ & Number of trains & $\begin{array}{c}\text { Primal problem } \\
\text { target value }\end{array}$ & $\begin{array}{c}\text { Dual problem } \\
\text { target value }\end{array}$ & Absolute gap & $\begin{array}{c}\text { Relative } \\
\text { gap (\%) }\end{array}$ & $\begin{array}{c}\text { Calculatio } \\
\mathrm{n} \text { time } \\
\text { (seconds) }\end{array}$ \\
\hline \multirow{3}{*}{$\begin{array}{c}\text { Subgradien } \\
\text { t method }\end{array}$} & 64 pairs & 77,286 & 79,710 & 2,424 & $3.14 \%$ & 42 \\
\cline { 2 - 7 } & 89 pairs & 98,346 & 110,863 & 12,517 & $12.73 \%$ & 60 \\
\cline { 2 - 7 } & 111 pairs & 122,066 & 136,570 & 14,504 & $11.88 \%$ & 51 \\
\hline \multirow{3}{*}{$\begin{array}{c}\text { Bundle pairs } \\
\text { method }\end{array}$} & 151,674 & 179,404 & 27,730 & $18.3 \%$ & 67 \\
\cline { 2 - 7 } & 84 pairs & 78,062 & 79,368 & 1,576 & $2.01 \%$ & 45 \\
\cline { 2 - 7 } & 111 pairs & 101,084 & 104,423 & 3,339 & $3.30 \%$ & 112 \\
\hline
\end{tabular}

The results show that the solutions are close to the optimal solution and the convergence speed is good. As we consider the station tracks and sections rather than running routes as competing resources, the model is actually a macroscopic model. The conflicts and searching space in our model are rather limited compared to a microscopic model, but our model is suitable for many HSR lines because the throat layout of HSR stations is rather simple.

As shown in Table 4, in each of the four cases, duality gaps of less than $20 \%$ were achieved when the subgradient method was used; however, the duality gaps obtained using the bundle method were all less than $8 \%$. We therefore conclude that LR using the bundle method is more efficient and can yield a more accurate solution, although this approach requires a longer calculation time. And train timetables of improved quality can be obtained within a short computation time by applying the LR algorithm.

\section{Conclusions}

In the case study of a real-world railway line, the Beijing-Shanghai high-speed railway, we obtain an improved solution compared with the current timetable, with a smaller duality gap (less than $8 \%$ ), in a short amount of time. In certain cases, our heuristic can rapidly produce a better timetable and schedule more trains in a real-world scenario compared with previously developed methodologies.

\section{Acknowledgements}

This work was financially supported by the Technology Research and Development Program of China railway (2017X009-E).

\section{References}

[1] A. A. Assad, Models for rail transportation, Transportation Research Part A: General, vol. 14, no. 3, 1980, pp. 205-220. 
[2] J. F. Cordeau, P. Toth and D. Vigo, A survey of optimization models for train routing and scheduling, Transportation science, vol. 32, no. 4, 1998, pp. 380-404.

[3] I. A. Hansen, Railway network timetabling and dynamic traffic management, International Journal of Civil Engineering, vol. 8, no. 1, 2010, pp. 19-32.

[4] V. Cacchiani and P. Toth, Nominal and robust train timetabling problems, European Journal of Operational Research, vol. 219, no. 3, 2012, pp. 727-737.

[5] F. Corman, A. D'Ariano, D. Pacciarelli and M. Pranzo, Bi-objective conflict detection and resolution in railway traffic management, Transportation Research Part C: Emerging Technologies, vol. 20, no. 1, 2012, pp. 79-94.

[6] M. Carey and I. Crawford, Scheduling trains on a network of busy complex stations, Transportation Research Part B: Methodological, vol. 41, no. 2, 2007, pp. 159-178.

[7] B. Adenso-Diaz, M. O. González and P. González-Torre, On-line timetable re-scheduling in regional train services, Transportation Research Part B: Methodological, vol. 33, no. 6, 1999, pp. 387-398.

[8] İ. Şahin, Railway traffic control and train scheduling based oninter-train conflict management, Transportation Research Part B: Methodological, vol. 33, no. 7, 1999, pp. 511-534.

[9] Yue, Yixiang, et al. "Optimizing train stopping patterns and schedules for high-speed passenger rail corridors." Transportation Research Part C: Emerging Technologies 63 ,2016, pp. 126-146. 